\title{
Enhanced Bandwidth Nonlinear Resonance Electromagnetic Human Motion Energy Harvester Using Magnetic-Springs and Ferrofluid
}

\author{
Chunfang Li, Shuai Wu, P. C. K. Luk, Min Gu, and Zongxia Jiao
}

\begin{abstract}
An enhanced bandwidth nonlinear resonant electromagnetic energy harvester has been designed to harness low frequency energy from basic human motion. Some vertical stacked cylindrical permanent magnets (PMs) constitute the inertial mass of the proposed harvester, which is suspended axially by two magnetic-springs and circumferentially by ferrofluid within a carbon fiber tube. In order to widen the frequency band and improve harvesting efficiency, two PMs are respectively fixed on the two end caps of the carbon fiber tube, so as to form two magnetic-springs with variable stiffness by cooperating with the PM stack. The self-assembled ferrofluid around the PM stack acts as its bearing system to minimize any friction during its movement. Copper wire are wrapped outside the tube to form the armature winding. The stiffness characteristic of the magneticsprings and the optimum equilibrium position and number of windings have been determined by finite element method (FEM) analysis. As a proof of concept, a portable prototype of the proposed energy harvester that weighs $110 \mathrm{~g}$ and with a volume of only $37.7 \mathrm{~cm}^{3}$ is fabricated. A series of experiments are carried out and the results show that the frequency band of the harvester becomes wider as the external vibration intensity increases. In addition, the effectiveness of ferrofluid in reducing friction is demonstrated under walking and running conditions. Without ferrofluid, the maximum average outputs are $10.15 \mathrm{~mW}$ and $32.53 \mathrm{~mW}$ respectively for walking and running. With ferrofluid, the maximum outputs are $17.72 \mathrm{~mW}$ and $54.61 \mathrm{~mW}$, representing an increase of $74.58 \%$ and $67.88 \%$, respectively. Furthermore, the prototype exhibits an average power density of $1.45 \mathrm{~mW} / \mathrm{cm}^{3}$ during running motions, which compares favorably with existing harvesters used in low power wearable devices.
\end{abstract}

Index Terms-Nonlinear resonance, vibration energy harvester, human motion, magnetic-spring, ferrofluid

\section{INTRODUCTION}

W ITH the development of technology, portable and wearable electronic devices are gradually reaching into people's lives, such as mobile phones, smart watches and wearable cameras. The energy supply for these portable and wearable electronic devices comes mainly from batteries. Despite great advances in battery technology, battery capacity

Manuscript received Month xx, 2xxx; revised Month xx, xxxx; accepted Month X, xxxx.

Shuai $\mathrm{Wu}$ is with the School of Automation Science and Electrical Engineering, Beihang University, Beijing, 100191 China (e-mail: wushuai.vip@gmail.com), and also with the State Key Laboratory of Fluid Power and Mechatronic Systems, Zhejiang University, Hangzhou. 310027, China.

Chunfang Li, Min Gu and Zongxia Jiao are with the School of Automation Science and Electrical Engineering, Beihang University, Beijing, 100191 China.

P. C. K. Luk is with the Electrical Power and Drives Group, Power Engineering Centre, Cranfield University, Cranfield, MK43 0AL, U.K is still a feature that limits the long-term working. On the other hand, charging these portable and wearable devices under some conditions, e.g. long time field, is inconvenient or impossible. Indeed, human motion contains sufficient energy for these devices. Therefore, it is of great significance to develop a small energy harvester which can harvesting human motion energy and charging the battery continuously. And this idea has attracted a lot of research interesting recently. In general, the harvesting of human motion energy is achieved through various transfer mechanisms such as piezoelectric [1][3], electrostatic [4]-[6], electromagnetic [7]-[15], and hybrid [16], [17], which convert human motion energy into electrical energy [18]. However, the piezoelectric energy harvesters has a disadvantage of low generation efficiency in low frequency and electrostatic energy harvesters require very high processing technologies. Therefore, the electromagnetic mechanism is chosen in this study because of the simple mechanical structure, suitable for low frequency motion and low cost.

The electromagnetic harvester usually using a resonance vibrator to generate electrical power [19]. For human motion, such as ankle swing and heel landing during walking and running, vibrations are generated that are mainly composed of frequencies of $\leq 10 \mathrm{~Hz}$ [20]-[22]. Therefore, energy harvesters that designed based on a specific resonant frequency appear to be less practical in vibration applications with a wide frequency range. In recent years, the use of various methods to broaden the harvestable frequency bandwidth of energy harvesters has extensively been reported as an alternative [23][27]. For example, Wei et al. [28] achieves a nonlinear resonant frequency by adjusting a magnetic-spring and the mass of the moving stack. Our group has been developed some human body energy harvesters designed by electromagnetic resonance to improve energy harvesting efficiency by using 3-dimensional resonance [29]. Additionally, the mechanical damping is a great challenge to design this type of small wearable electromagnetic energy harvesters, where friction is one of the important factors that results in their low power output. Therefore, new and innovative design solutions are needed to improve the performance of electromagnetic harvesters.

In the present study, a enhanced bandwidth nonlinear resonance electromagnetic energy harvester using magneticsprings and ferrofluid is proposed to harvest energy from human motion. The vibrator of the proposed harvester is a PM stack which has several PM rings are placed in opposing 
orientations and alternate with soft iron. The vibrator is centered in the axial direction by two PM rings which is placed at two end of the tube. The PM stack and two magnets at two ends forms a magnetic-springs which has nonlinear repulsive force. Due to the nonlinearity of the spring, the vibrator can experience resonance even when the excitation frequency is away from the natural frequency of the system which is very suit for human motion which has wide bandwidth of frequency in range of $0-10 \mathrm{~Hz}$. Ferrofluid is introduced which is adsorbed by PMs and fill the gap between PM stack and tube, and works like a hydrostatic bearing which reduce the friction force significantly. The use of nonlinear springs and ferrofluid improve the harvesting efficiency. A model of the harvester was built in Ansoft Maxwell software and its magnetic field was simulated by FEM method. Based on the results of FEM analysis and dynamic simulation, the parameters of the structure and windings are optimized. Then a fabricated prototype of the harvester is carried out and tested. The results indicate that output power density is higher compared with existing systems. The influence of ferrofluid and human motion patterns on the energy harvesting efficiency are investigated as well.

\section{Structural AND Operation PRINCIPLE}

The schematic and design diagram of the proposed electromagnetic energy harvester are shown in Fig. 1. It consists of a hollow tube with two permanent magnets fixed at two ends and an vibrator is that can move along the tube. The vibrator consists of four PM rings and alternate with three iron rings. The pole direction of PMs are along the tube and placed in opposing orientations to each adjacent ones. A hollow tube is used to fix the vibrator stack and the pipe allow to connect two cavities at both ends during the PM stack movement. The vibrator is suspended in the equilibrium position due to the repulsive forces of the magnetic-springs when no external force. Three winding rings are wound over the outer surface of the tube and the flux of the PMs pass through the winding. When the harvester is subjected to external excitation, the vibrator will vibrate along the tube, causing magnetic flux to be cut by windings to generate electrical energy through electromagnetic induction. Assuming the vibrator moves upward, the induced current of each winding will be shown as Fig. 1. The transducer mechanism of an electromagnetic energy harvester is based on Faradays law of electromagnetic induction which states that the voltage generated $U$ is related to the rate of change of net magnetic flux [30]. The voltage generated $U_{\mathrm{m}}$ across a winding can be given by

$$
U_{\mathrm{m}}=-n \frac{d \phi}{d t}=-n \frac{d\left(\int \vec{B} \cdot d \vec{A}\right)}{d t}=-n B l \dot{z}
$$

where $n$ is the number of windings turns, $\dot{z}$ is relative velocity between PM and the winding, $\phi$ is the magnetic flux, $B$ is the magnetic flux density, $d \vec{A}$ is the differential element area of a magnet-winding assembly, $l$ is the total length of windings.

Two PMs are placed at two ends of the tube and the pole orientation of each PM is opposite to the faced end of PM stack to form a magnetic-spring. Since the repulsive force of magnetic-spring is non-linearity with the distance of the vibrator to the fixed ends, the harvester is a nonlinear harmonic vibration system that is very suitable for human energy harvest which the detail will be discussed in the next sections.

The friction between vibrator and tube will deplete most the energy of the harvester. In order to improve the efficiency of the harvester, ferrofluid is introduced which can be absorbed around the PM like hydrostatic bearings to make the vibrator levitated away from the inner wall of the tube which make the friction extremely small.

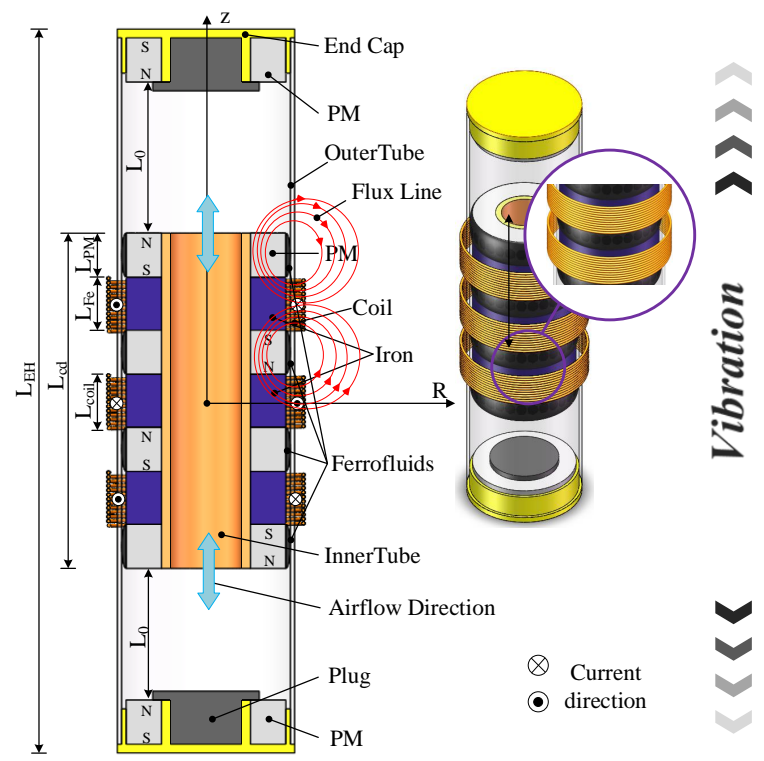

Fig. 1: The structure of the proposed energy harvester

\section{Optimization B Y MODEling AND Simulation}

The harvester should be optimized to get maximum output power and efficiency. There are a lot of parameters should be decided. In the present study, the parameters of PM stack and tube are predefined which is shown in Table. I and the parameters of magnetic-spring and winding are optimized according to the predefined parameters.

TABLE I: Predefined parameters of the energy harvester.

\begin{tabular}{lll}
\hline Parameter & Symbol & Value \\
\hline Inner diameter of tube & $d_{\mathrm{tr}}$ & $19 \mathrm{~mm}$ \\
Outer diameter of tube & $D_{\mathrm{to}}$ & $20 \mathrm{~mm}$ \\
Inner diameter of PM & $d_{\mathrm{mr}}$ & $10 \mathrm{~mm}$ \\
Outer diameter of PM & $D_{\mathrm{mo}}$ & $18 \mathrm{~mm}$ \\
Thickness of the PM & $L_{\mathrm{PM}}$ & $5 \mathrm{~mm}$ \\
Thickness of the iron & $L_{\mathrm{iron}}$ & $6 \mathrm{~mm}$ \\
Length of the PM stack & $L_{\mathrm{stack}}$ & $38 \mathrm{~mm}$ \\
Mass of the vibrator & $M$ & $70 \mathrm{~g}$ \\
\hline
\end{tabular}

\section{A. Magnetic Spring Optimization}

A schematic diagram of the proposed nonlinear resonant electromagnetic harvester is shown in Fig. 2. The harvester can be regarded as a nonlinear spring-damping vibrator system 
that is excited by external motion. The dynamic model of the motion can be expressed as [31], [32]:

$$
m z \ddot{(t})+\left(c_{m}+c_{e}\right) \dot{z(t)}+F_{r}(t)=m \ddot{y}(t)
$$

where $m$ is the mass of PM stack, the absolute displacement of PM stack and the frame are $x(t)$ and $y(t)$, respectively, the relative displacement of the PM stack can be got by $z(t)=$ $x(t)-y(t), F_{r}$ is the nonlinear repulsive force of the moving magnetic stack, $c_{m}$ is the mechanical damping coefficient and $c_{e}$ is the electrical damping coefficient.

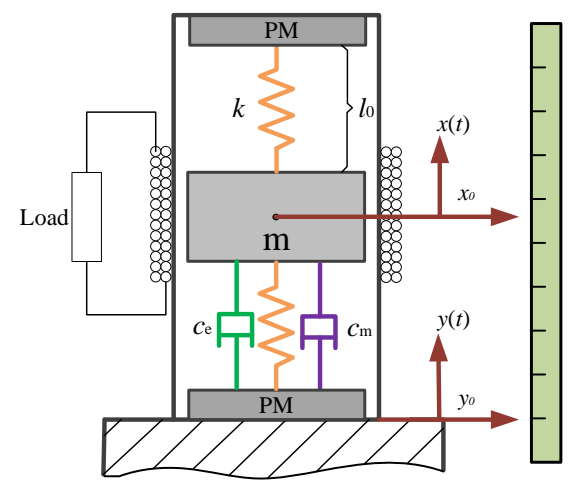

Fig. 2: Schematic diagram of energy harvester model

The initial distance $\left(l_{0}\right)$ between the end PM and PM stack is a key parameter in determining the stiffness of the magneticspring. In this study, this distance is optimized by the FEA simulation and dynamic simulation. The repulsive force $F_{r}$ of the magnetic-spring has a nonlinear relationship with the relative distance $z$, which can be expressed as [28]

$$
F_{r}=k_{1} z+k_{2} z^{3}
$$

where $k_{1}$ and $k_{2}$ are polynomial coefficients of the repulsive force. It is a typical periodically forced oscillator with the nonlinear elasticity, which can be written as

$$
\ddot{z}+2 \xi \omega_{n} \dot{z}+\omega_{n}^{2} z+\beta z^{3}=F \cos (\omega t)
$$

where $2 \xi \omega_{n}=c / m, c=c_{e}+c_{m}, \omega_{n}^{2}=k_{1} / m, \beta=k_{2} / m$, $F=-\omega^{2} A$.

It is usually called a duffing oscillator which has more complex potential than in simple harmonic motion. For a certain range of parameters in the Eq. 4, the frequency response may no longer be a single-valued function of forcing frequency $\omega$. Since magnetic-spring is like a hardening spring $\left(k_{1}>0\right.$ and $k_{2}>0$ ), the frequency response will hang to the high frequency side.

The FEA simulation results of $F_{r}$ with $l_{0}=15,17,19 \mathrm{~mm}$ are shown in Fig. 3a. And the value of $k_{1}$ and $k_{2}$ under different $l_{0}$ are also obtained by polynomial fitting. Thus the coefficients of $k_{1}$ and $k_{2}$ are lie on $l_{0}$. The frequency response with different $l_{0}$ is obtained by dynamic simulation is shown in Fig. 3b. The Gray area is the expected frequency range $0-6 \mathrm{~Hz}$ and the amplitude $z$ is $6 \mathrm{~mm}$. As can be seen in Fig. 3b, when $l_{0}=17 \mathrm{~mm}$, it can best meet the frequency range of human motion, so that the output power generated by the harvester

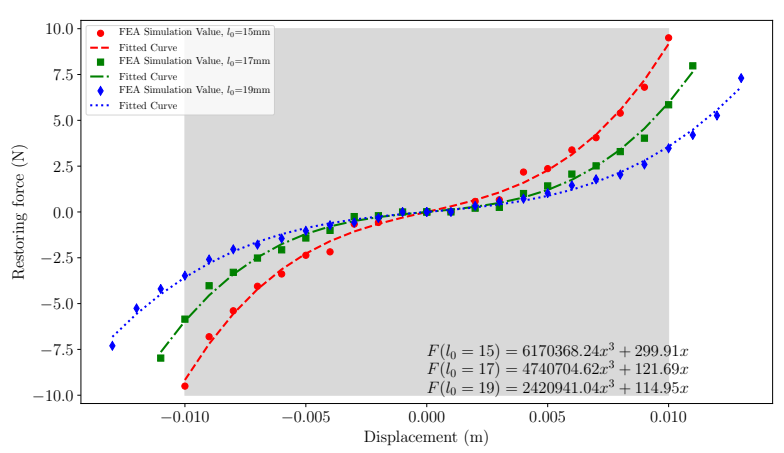

(a) Nonlinear repulsive force.

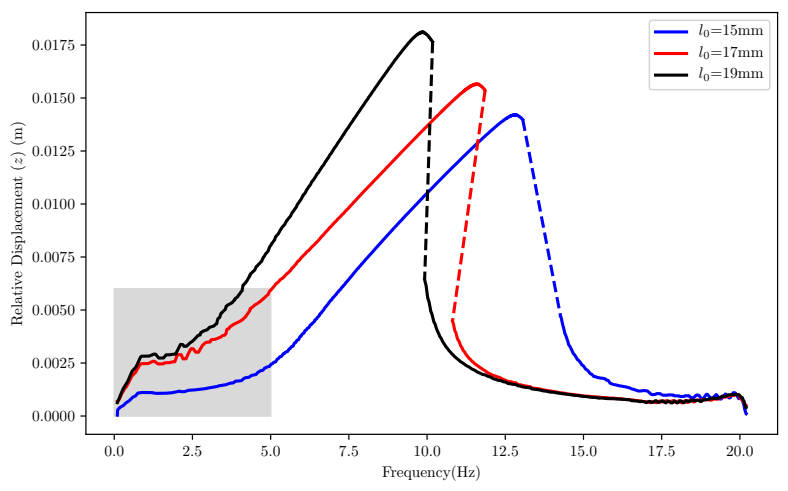

(b) Frequency response of the relative displacement

Fig. 3: The dynamic simulation results of harvester

is the maximum. Therefore, the $l_{0}=17 \mathrm{~mm}$ is selected in the present study.

\section{B. Magnetic field simulation and windings design}

To achieve maximum output power, windings also needs to be optimized based on the magnetic field of the PM stack. The most important parameters of a winding include the diameter of the enameled wire $\left(d_{c}\right)$, the number of winding layer $(n)$ and the length of a winding $\left(L_{\text {coil }}\right)$. These three parameters decide the total length and resistance of the enameled wire used. The total length of the enameled wire $(l)$ can be calculated as

$$
l=3 \sum_{i=1}^{n} \frac{L_{\text {coil }}}{d_{c}} 2 \pi\left(0.5 D_{\text {to }}+(n-0.5) d_{c}\right)
$$

And the resistance of windings can be calculated as

$$
R=\rho \frac{l}{s}=\rho \frac{4 l}{d_{c}^{2}}
$$

where $R$ is the resistance of windings, $\rho$ is the coefficient of enameled wire. The resistance of windings should match to the load impedance which should be decided with the magnetic field distribution.

The magnetic field distribution of PM stack is analyzed by FEM analysis. The simulation model and results are illustrated in Fig. 4. The ferrofluid does not considered in this FEM simulation since it is very easy saturated and have little effect 
on filed density. The simulation model is established based on the design parameters, the total height of PM stack is $38 \mathrm{~mm}$. The center radius of each layer in the winding also are marked with $r=10.15 \mathrm{~mm}, r=10.45 \mathrm{~mm}, r=10.75 \mathrm{~mm}$ and $r=11.05 \mathrm{~mm}$, respectively. As can be seen in Fig. 4, the magnetic flux lines between two magnets are guided by a pole piece that allows the flux lines to interact with the windings along the radial direction as best as possible, increasing the power output. Additionally, the magnetic flux density becomes smaller as the center radius $r$ of each layer increases.

The magnetic flux density in radial direction Mag_Br at different locations are shown in Fig. 5, where the horizontal axis represents the height of the PM stack. Fig. 5 shows that Mag_Br gets a maximum value in the gray range, where the gray ranges are the installation positions of iron pole pieces. Deviation from the gray range causes Mag_Br to decrease, and drop to $0 \mathrm{~T}$ at some certain positions. Therefore, the maximum voltage can be generated from a winding when the winding corresponds to the position of the pole pieces. For purpose of high output power, three windings are wound at positions corresponding to three pole pieces when the vibrator is at its equilibrium position. Of course, these positions are the optimum equilibrium positions of windings. Fig. 5 also exhibits Mag_Br at different values of $r$, and Mag_Br decreases as $r$ grows.
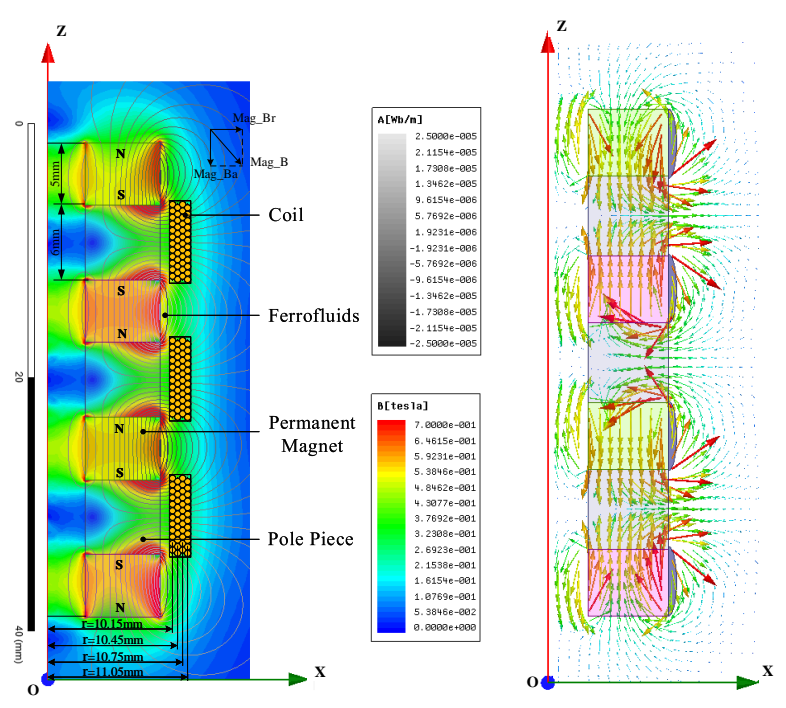

Fig. 4: The magnetic flux density distribution of PM stack

Then the winding parameters can be decided. In the present study, the load impedance is $4 \Omega$. $L_{\text {coil }}=6 \mathrm{~mm}$ is set for cover the maximum flux density range. The diameter of enameled wire and winding layer can be optimized by Eq. 5 and 6 . $d_{c}=0.3 \mathrm{~mm}$ and 4 layer is chosen in the present study.

\section{PROTOTYPE AND EXPERIMENT}

\section{A. Prototype and Test Rig}

The prototype of the proposed variable resonance electromagnetic energy harvester is fabricated and the entire test rig is shown in Fig. 6. The carbon fiber tube was chosen in this prototype because of its advantages of high structural strength,

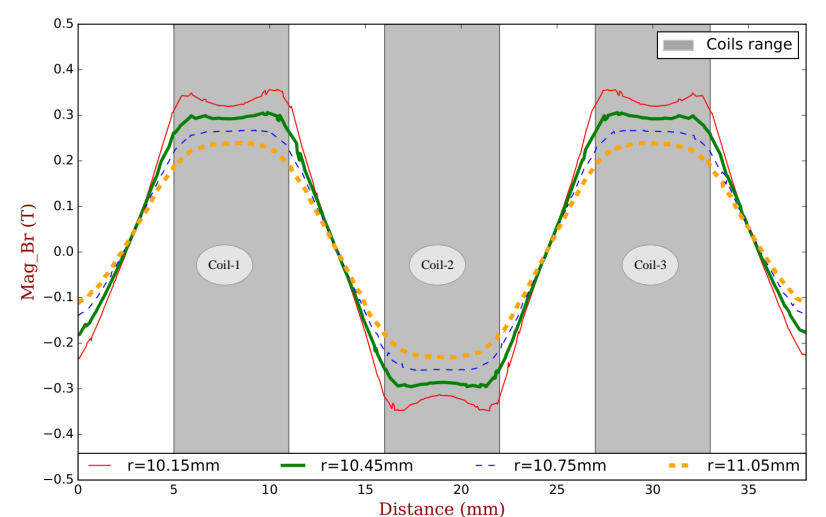

Fig. 5: Magnetic flux densities in radial direction of different radius

corrosion resistance and light weight. The PM stack, which works as a proof mass, consists of four $N d F e 35$ Magnets and three carbon steel hollow blocks. PM stack is suspended in the axial direction by the repulsive forces between PM stack and two magnets fixed at both ends of the carbon fiber tube. In the circumferential direction, PM stack is 'wrapped' by ferrofluid. The role of ferrofluid is to keep the PM stack away from the inner wall of carbon fiber tube, reducing the friction of PM stack during the movement. It is expected that the design resistance of the proposed energy harvester is about $4 \Omega$ to match an energy storage circuit being designed. The energy storage circuit will not be discussed in this paper. According to simulation results and actual requirements, it is decided that three windings made of $0.3 \mathrm{~mm}$ enamelled wire are wrapped around the outside of the carbon fiber tube at the optimum equilibrium positions of windings. As the number of winding layers increase, the increase in voltage generated by this winding may be less than the increase in the resistance of the winding, which in turn reduced the output power of the proposed harvester. Therefore, Each winding is made with 4 layers, measuring the resistance of each winding is $1.3 \Omega$, and the total resistance of the prototype is $3 \times 1.3 \Omega=3.9 \Omega$. After detecting and comparing the waveforms of the generated voltage from the three windings, they are then connected in series to achieve the maximum energy output. The full geometric parameters are also presented in Table. II.

The test rig mainly consists of a voice coil motor (LA3048-000A, BEI), a data acquisition module (NI USB-4431), a brush type PWM servo amplifier (AMC) and two computer. It is also clearly observed that the glistening ferrofluid covered the surface of PMs in Fig. 6, especially the edge of PM, where magnetic field density is the greatest.

\section{B. Experimental Results and Discussion}

In order to measure the effective frequency band of the prototype, the open circuit vibration test is carried out. The voice coil motor is driven to provide the required excitation frequency and different acceleration levels. The output voltage of prototype is obtained by the data acquisition module. The open circuit output voltages of the proposed energy 


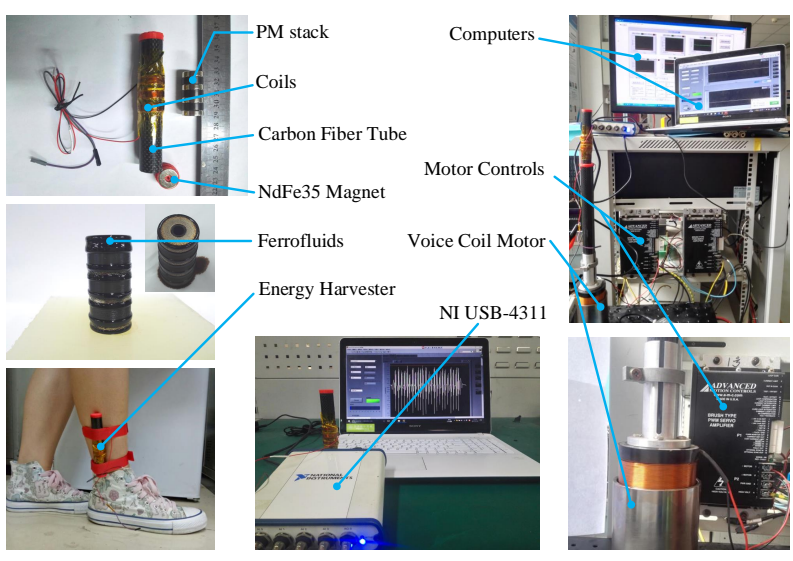

Fig. 6: The prototype of energy harvester, experimental devices and equipment

TABLE II: Optimized parameters of the energy harvester

\begin{tabular}{lll}
\hline Parameter & Symbol & Value \\
\hline Initial distance of PM spring & $l_{0}$ & $17 \mathrm{~mm}$ \\
Length of a winding & $L_{\text {coil }}$ & $6 \mathrm{~mm}$ \\
Diameter of enameled wire & $d_{c}$ & $0.3 \mathrm{~mm}$ \\
Winding layers & $n$ & 4 \\
Turns of three windings & $N$ & 240 \\
Total resistance of windings & $R_{i}$ & $3.9 \Omega$ \\
Total Mass of the harvester & $M$ & $110 \mathrm{~g}$ \\
Total Length of the harvester & $L_{\mathrm{EH}}$ & $120 \mathrm{~mm}$ \\
\hline
\end{tabular}

harvester at different excitation levels and different excitation frequencies are collected. The waveforms of the output voltage under different excitation conditions are shown in Fig. 7a, where the acceleration levels are set to $0.16 \mathrm{~g}, 0.20 \mathrm{~g}$ and $0.25 \mathrm{~g}$, the excitation frequency is set to $1 \mathrm{~Hz}, 2 \mathrm{~Hz}, 3 \mathrm{~Hz}$, $\cdots, 10 \mathrm{~Hz}$, and each excitation condition is applied for $2 \mathrm{~s}$. As can be seen in Fig. 7a, the output voltage generated by the prototype increases as the acceleration level increases at each excitation frequency. For an acceleration level of $0.16 \mathrm{~g}$, the generated maximum voltage is about $2 \mathrm{~V}$ at $6 \mathrm{~Hz}$. For a large acceleration of $0.20 \mathrm{~g}$, the voltages generated at the three excitation frequencies of $6 \mathrm{~Hz}, 7 \mathrm{~Hz}$ and $8 \mathrm{~Hz}$ are relatively higher. At an acceleration level of $0.25 \mathrm{~g}$, a maximum voltage of $5 \mathrm{~V}$ is achieved at a frequency of $9 \mathrm{~Hz}$. Fig. $7 \mathrm{~b}$ shows the RMS voltage of different excitation conditions. It can found that the effective bandwidth of the harvester becomes wider when the acceleration level increases. Therefore, the voltage response and the effective bandwidth exhibit a increasing trend with the increasing excitation levels, thanks to the nonlinear magnetic-spring.

Then, the output performance of the proposed harvester is evaluated from basic human motions including walking and running by fixing the harvester near to an ankle. The open circuit instantaneous voltage waveforms and frequency spectrum during walking and running are shown Fig. 8 and Fig. 9, respectively. The frequency of walking motion is about $1 \mathrm{~Hz}$, and running frequency is roughly $1.43 \mathrm{~Hz}$. As can be seen in these two figures, the instantaneous voltage generated by running is higher than the walking. Compared with the presence and absence of the ferrofluid experiments in the same motion mode, it is found that the voltage amplitude decay is slowed down after adding ferrofluid. And the sound generated by the friction between the PM stack and the carbon fiber inner wall also is weaken during the experiment. Therefore, it can be inferred that the ferrofluid plays an important role in reducing friction.

Fig. 10 shows the output RMS voltage and average power delivered to load $R_{o}$ range of from $1 \Omega$ to $10 \Omega$ by the prototype during the same walking and running style as above. As can be seen from these average power curves, the maximum output power corresponded to a load of $4 \Omega$. Additionally, it is noteworthy that more energy can be generated during running motion than walking motion. For walking motion, the value of RMS voltage delivered to the optimum load of $4 \Omega$ is $0.22 \mathrm{~V}$ without ferrofluid, while the use of ferrofluid increases by $0.29 \mathrm{~V}$. According to $\bar{P}=V_{r m s}^{2} / R_{o}$ calculates the average power in both cases are $10.15 \mathrm{~mW}$ and $17.72 \mathrm{~mW}$, respectively. Where $V_{r m s}$ is the RMS voltage across the corresponding load resistance $R_{o}$. And The latter relative to the former increases by around $74.58 \%$. For running motion, the output maximum average power of using ferrofluid are $54.61 \mathrm{~mW}$ from the experimentally obtained results, which is about $67.88 \%$ higher than the case where ferrofluid are not used. In summary, ferrofluid are indeed beneficial to improve the output power.

Table. III shows a summary of the proposed harvester and the previously reported electromagnetic energy harvester. The designed harvester has a small volume, a simple structure, a low manufacturing cost and convenient carrying, but it still needs to be further improved in power generation capacity.

\section{CONCLUSions}

An nonlinear resonance electromagnetic energy harvester using magnetic-springs and ferrofluid for suspension is presented in the present study. This energy harvester is designed to scavenge energy from human motions to supply power for wearable devices. This harvester adopts two magneticsprings with a stiffness varying with vibration displacement to widen the frequency band, thereby increasing the generated energy. Additionally, ferrofluid reduces the friction of the proposed energy harvester and makes it highly efficient in producing electrical power. The characteristics of magneticspring, the optimum equilibrium position of windings and number of windings also are determined by FEM analysis in Ansoft Maxwell. The prototype of the proposed energy harvester that weighs $110 \mathrm{~g}$ and with a volume of only $37.7 \mathrm{~cm}^{3}$ has been fabricated, and a range of experiments are carried out to characterize the proposed harvester. The experimental results of the open circuit frequency response show that the frequency band of the harvester widens as excitation intensity increases. Furthermore, the effectiveness of the ferrofluid in reducing friction is demonstrated under walking and running conditions. Two maximum average output power of $17.72 \mathrm{~mW}$ and $54.61 \mathrm{~mW}$ are obtained by the proposed energy harvester of using ferrofluid from walking and running. Compared to the case of the absence of ferrofluid, the outpus are increased by $74.58 \%$ and $67.88 \%$, respectively. In the future work, the size 
TABLE III: Summary of this work with previously reported electromagnetic energy harvester

\begin{tabular}{lllllll}
\hline References & Frequency $(\mathrm{Hz})$ & $\begin{array}{l}\text { Average } \\
(\mathrm{mW})\end{array}$ & Power & Volume $\left(\mathrm{cm}^{3}\right)$ & Mass $(\mathrm{g})$ & $\begin{array}{l}\text { Power } \\
\left(\mathrm{mW} / \mathrm{cm}^{3}\right)\end{array}$ \\
\hline Miah A. Halim et al. [7] & 5.17 & 2.15 & 6.47 & 18.6 & 0.33 & $\begin{array}{l}\text { Density } \\
\text { Condition }\end{array}$ \\
Qian Zhang et al. [14] & 5.5 & 14.8 & 26 & 98 & 0.57 & Hand-shaken \\
Wei Wang et al. [28] & - & 10.66 & 47 (Estimate) & 218.7 & 0.227 & Slow running \\
Ylli K et al. [20] & - & 4.13 & 48 & - & 0.086 & Heg swing \\
Qian Zhang et al. [33] & 4 & 32 & 120 & 180 & 0.27 & Slow running \\
Jing Qiu et al. [34] & 12.65 & 90.35 & 164.27 & - & 0.55 & Vibration shaker \\
This work & - & 54.61 & 37.7 & 110 & 1.45 & Running \\
\hline
\end{tabular}

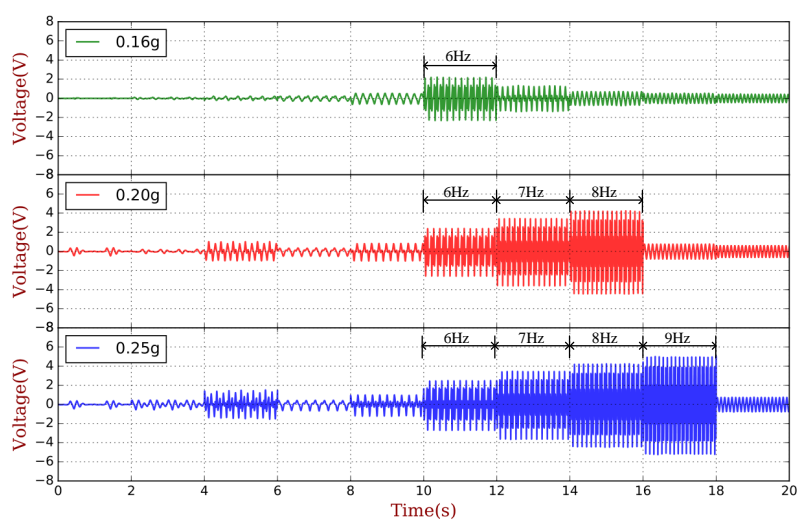

(a) Waveforms of different excitaciton frequency at different acceleration level

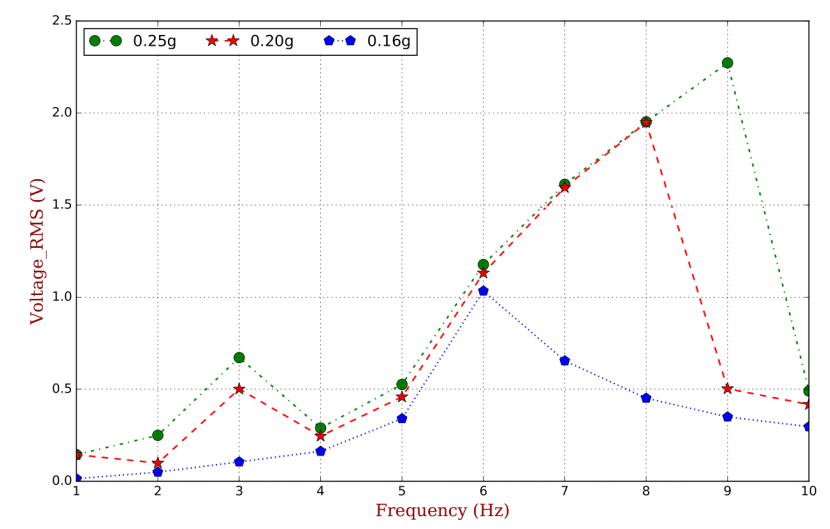

(b) The RMS voltage of different excitaciton frequency at different acceleration level

Fig. 7: Bandwidth of the proposed harvester under different acceleration level

of the pole pieces and the windings will be further optimized to achieve a higher energy conversion efficiency. Besides, an energy storage circuit that matches the proposed energy harvester also will be designed for better using in portable and wearable devices.

\section{ACKNOWLEDGMENT}

This work has been supported by the National Natural Science Foundation of China under Grant 51775014, the Beijing Natural Science Foundation under Grant 3192019, and
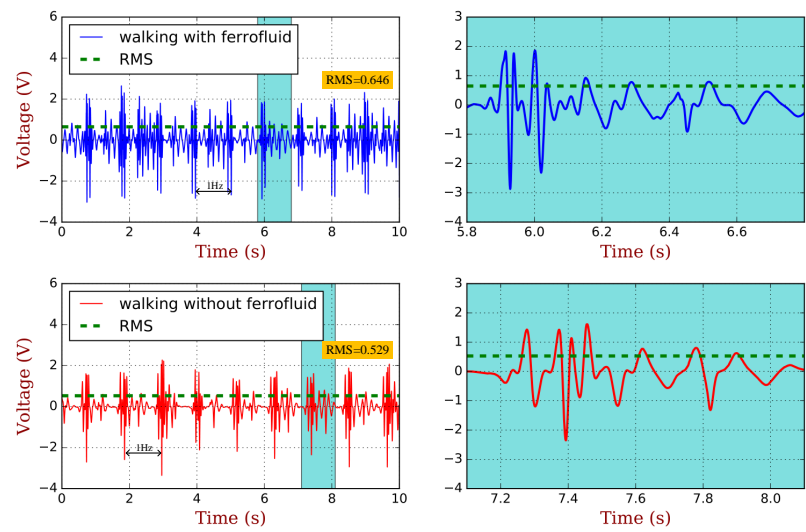

Fig. 8: Generated voltage waveforms during walking
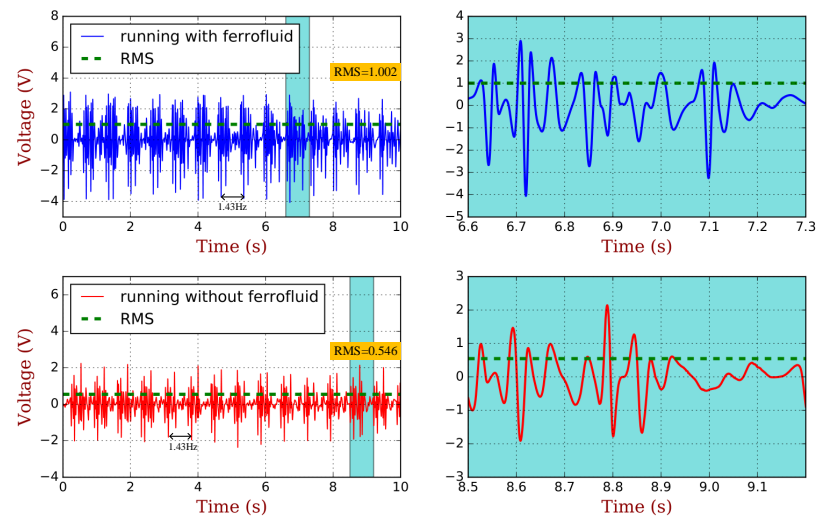

Fig. 9: Generated voltage waveforms during running

Open Foundation of the State Key Laboratory of Fluid Power and Mechatronic Systems.

\section{REFERENCES}

[1] X. Zheng, Z. Zhang, Y. Zhu, J. Mei, S. Peng, L. Li, and Y. Yu, "Analysis of energy harvesting performance for $d_{15}$ mode piezoelectric bimorph in series connection based on timoshenko beam model," IEEE/ASME Transactions on Mechatronics, vol. 20, no. 2, pp. 728-739, 2015.

[2] G. Bassani, A. Filippeschi, and E. Ruffaldi, "Human motion energy harvesting using a piezoelectric mfc patch," in 2015 37th Annual International Conference of the IEEE Engineering in Medicine and Biology Society (EMBC), 2015, pp. 5070-5073.

[3] J. Zhao and Z. You, "A shoe-embedded piezoelectric energy harvester for wearable sensors," Sensors, vol. 14, no. 7, pp. 12 497-12 510, 2014. 

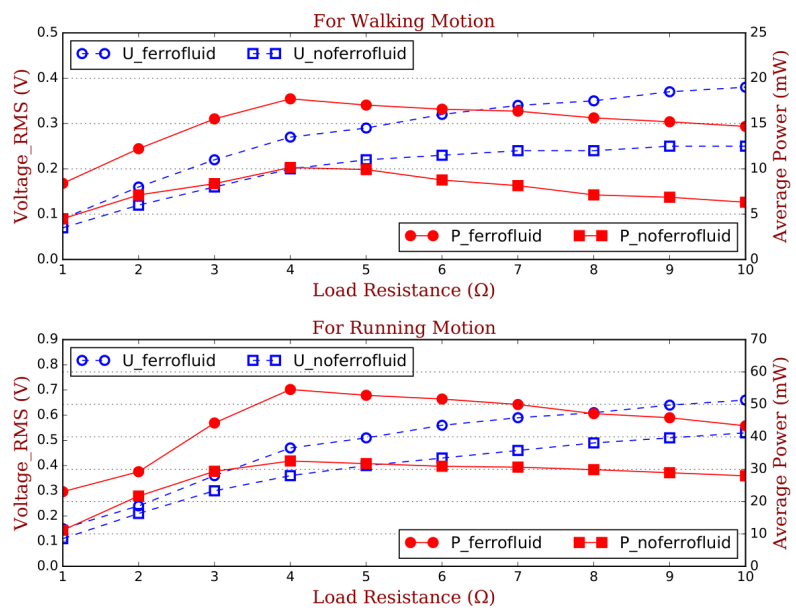

Fig. 10: Generated RMS voltage and average power delivered to various load resistances by the prototype during walking and running

[4] T. Galchev, R. Raz, and O. Paul, "An electrostatic springless inertial harvester for converting multi-dimensional low-frequency motion," in 2013 IEEE 26th International Conference on Micro Electro Mechanical Systems (MEMS), 2013, pp. 102-105.

[5] D. H. Choi, C. H. Han, H. D. Kim, and J. B. Yoon, "Liquid-based electrostatic energy harvester with high sensitivity to human physical motion," Smart Materials \& Structures, vol. 20, no. 12, p. 125012, 2011.

[6] Y. Lu, F. Cottone, S. Boisseau, F. Marty, D. Galayko, and P. Basset, "A nonlinear mems electrostatic kinetic energy harvester for humanpowered biomedical devices," Applied Physics Letters, vol. 107, no. 25 , pp. 429-2244, 2015.

[7] M. A. Halim, H. Cho, and J. Y. Park, "Design and experiment of a human-limb driven, frequency up-converted electromagnetic energy harvester," Energy Conversion \& Management, vol. 106, pp. 393-404, 2015.

[8] P. Podder, A. Amann, and S. Roy, "Combined effect of bistability and mechanical impact on the performance of a nonlinear electromagnetic vibration energy harvester," IEEE/ASME Transactions on Mechatronics, vol. 21, no. 2, pp. 727-739, April 2016.

[9] E. Bonisoli, M. Repetto, N. Manca, and A. Gasparini, "Electromechanical and electronic integrated harvester for shoes application," IEEE/ASME Transactions on Mechatronics, vol. 22, no. 5, pp. 19211932, Oct 2017

[10] F. Khan, F. Sassani, and B. Stoeber, "Copper foil-type vibrationbased electromagnetic energy harvester," Journal of Micromechanics \& Microengineering, vol. 20, no. 12, p. 125006, 2010.

[11] A. Bibo, R. Masana, A. King, G. Li, and M. F. Daqaq, "Electromagnetic ferrofluid-based energy harvester," Physics Letters A, vol. 376, no. 32, pp. 2163-2166, 2012.

[12] M. A. Halim, H. Cho, M. Salauddin, and J. Y. Park, "A miniaturized electromagnetic vibration energy harvester using flux-guided magnet stacks for human-body-induced motion," SENSORS AND ACTUATORS A-PHYSICAL, vol. 249, pp. 23-31, OCT 12016.

[13] X. Tang, T. Lin, and L. Zuo, "Design and optimization of a tubular linear electromagnetic vibration energy harvester," Mechatronics IEEE/ASME Transactions on, vol. 19, no. 2, pp. 615-622, 2014.

[14] Q. Zhang, Y. Wang, and E. S. Kim, "Electromagnetic energy harvester with flexible coils and magnetic spring for 1-10 hz resonance," Journal of Microelectromechanical Systems, vol. 24, no. 4, pp. 1193-1206, 2015.

[15] Y. Wang, Q. Zhang, L. Zhao, and E. S. Kim, "Non-resonant electromagnetic broad-band vibration-energy harvester based on self-assembled ferrofluid liquid bearing," Journal of Microelectromechanical Systems, vol. PP, no. 99, pp. 1-11.

[16] M. L. Seol, S. B. Jeon, J. W. Han, and Y. K. Choi, "Ferrofluidbased triboelectric-electromagnetic hybrid generator for sensitive and sustainable vibration energy harvesting," Nano Energy, vol. 31, pp. 233238, 2017.

[17] W. Yang and S. Towfighian, "A hybrid nonlinear vibration energy harvester," Mechanical Systems \& Signal Processing, vol. 90, pp. 317 333, 2017.

[18] M. Perton, B. Audoin, Y. D. Pan, and C. Rossignol, "Energy harvesting vibration sources for microsystems applications," Measurement Science \& Technology, vol. 17, no. 12, pp. R175-R195, 2006.

[19] S. Wu, P. C. K. Luk, C. Li, X. Zhao, and Z. Jiao, "Investigation of an electromagnetic wearable resonance kinetic energy harvester with ferrofluid," IEEE Transactions on Magnetics, vol. PP, no. 99, pp. 1-1, 2017.

[20] K. Ylli, D. Hoffmann, A. Willmann, P. Becker, B. Folkmer, and Y. Manoli, "Energy harvesting from human motion: exploiting swing and shock excitations," Smart Materials and Structures, vol. 24, no. 2, p. $025029,2015$.

[21] M. Gorlatova, J. Sarik, G. Grebla, M. Cong, I. Kymissis, and G. Zussman, "Movers and shakers: Kinetic energy harvesting for the internet of things," Selected Areas in Communications, IEEE Journal on, vol. 33, no. 8, pp. 1624-1639, 2015.

[22] G. V. Merrett, H. Huang, and N. M. White, "Modeling the effect of orientation on human-powered inertial energy harvesters," IEEE Sensors Journal, vol. 15, no. 1, pp. 434-441, Jan 2015.

[23] L. Tang, Y. Yang, and C. K. Soh, "Toward broadband vibration-based energy harvesting," Journal of Intelligent Material Systems \& Structures, vol. 21, no. 18, pp. 1867-1897, 2010.

[24] B. C. Lee and G. S. Chung, "Frequency tuning design for vibrationdriven electromagnetic energy harvester," Iet Renewable Power Generation, vol. 9, no. 7, pp. 801-808, 2015.

[25] J. Chen, D. Chen, T. Yuan, and X. Chen, "A multi-frequency sandwich type electromagnetic vibration energy harvester," Applied Physics Letters, vol. 100, no. 21, p. 1257, 2012.

[26] Z. Yang, J. Zu, and Z. Xu, "Reversible nonlinear energy harvester tuned by tilting and enhanced by nonlinear circuits," IEEE/ASME Transactions on Mechatronics, vol. 21, no. 4, pp. 2174-2184, 2016.

[27] B. L. Ooi, J. M. Gilbert, and A. R. A. Aziz, "Switching damping for a frequency-tunable electromagnetic energy harvester," Sensors \& Actuators A Physical, vol. 234, pp. 311-320, 2015.

[28] W. Wang, J. Cao, N. Zhang, J. Lin, and W.-H. Liao, "Magnetic-spring based energy harvesting from human motions: Design, modeling and experiments," Energy Conversion and Management, vol. 132, pp. 189 197, 2017.

[29] S. Wu, P. Luk, C. Li, X. Zhao, Z. Jiao, and Y. Shang, "An electromagnetic wearable 3-dof resonance human body motion energy harvester using ferrofluid as a lubricant," Applied Energy, vol. 197, pp. 364-374, 2017.

[30] M. Mizuno and D. G. Chetwynd, "Investigation of a resonance microgenerator," Journal of Micromechanics \& Microengineering, vol. 13, no. 2, p. 209, 2003.

[31] M. Masoumi and Y. Wang, "Repulsive magnetic levitation-based ocean wave energy harvester with variable resonance: Modeling, simulation and experiment," Journal of Sound \& Vibration, vol. 381, pp. 192-205, 2016.

[32] B. P. Mann and N. D. Sims, "Energy harvesting from the nonlinear oscillations of magnetic levitation," Journal of Sound \& Vibration, vol. 319 , no. 12, pp. 515-530, 2009.

[33] Y. Zhang Qian, Wang and E. S. Kim, "Power generation from human body motion through magnet and coil arrays with magnetic spring," Journal of Applied Physics, vol. 115, no. 6, p. 083105, 2014.

[34] J. Qiu, X. Liu, Y. Wen, P. Li, and W. Li, "A low-frequency resonant electromagnetic vibration energy harvester employing the halbach arrays for intelligent wireless sensor networks," in Magnetics Conference, 2015, pp. $1-1$.

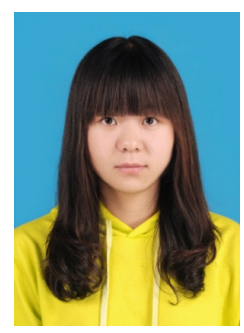

Chunfang Li Chunfang Li was born in ShanXi, China in 1993. She received a bachelor's degree from the Department of Mechanical Engineering, North China Electric Power University (Baoding), in 2016. She is currently studying for a master's degree in the School of Automation Science and Electrical Engineering at Beihang University. Her research interests include mechatronics, modeling and simulation. 


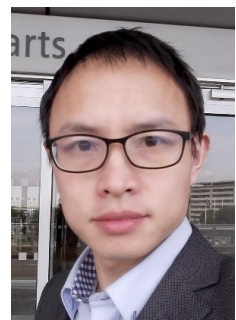

Shuai Wu ShuaiWu (M'15) received the Ph.D. degree in mechatronics engineering from Beihang University, Beijing, China, in 2011. He was with Beihang University, Beijing, China, as a Postdoctoral Fellow from 2011 to 2013 and has been a Lectuer there since 2013. His research interests include hydraulic servo control of aircraft, modeling and simulation, dynamics and control ofmechatronic systems, and advanced electrical, and hydraulic servo components.

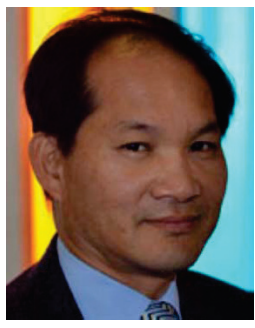

Patrick Chi-Kwong Luk Patrick Chi-Kwong Luk (SM10) was born in Hong Kong. He received the High Diploma degree with merits from Hong Kong Polytechnic University (PolyU), Hung Hom, Hong Kong, in 1983, the M.Phil. degree from Sheffield University, Sheffield, U.K., in 1989, and the Ph.D. degree from the University of South Wales, Newport, U.K., in 1992, all in electrical engineering. He started his career in industry as an Engineer Trainee between 1981 and 1983 at GEC (H.K.) and then after graduation as an Applications Engineer at Polytek Engineering Co. (H.K.). In 1986, he was a Senior Researcher in the Industrial Centre at PolyU. Beginning in 1988, he held academic positions at the University of SouthWales, Robert Gordon University, U.K., and the University of Hertfordshire, U.K. He joined Cranfield University, Cranfield, U.K., in 2002, where he is a Chair Professor in electrical engineering. His current research interests include electrical drives, renewable energy conversion. $\mathrm{He}$ has authored over 170

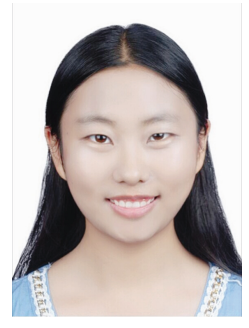

Min Gu Min Gu was born in Inner Mongolia, China in 1993. She received a bachelor's degree from the Department of Mechanical Engineering, Nanjing University of Aeronautics and Astronautics, in 2016. She is currently studying for a master's degree in the School of Automation Science and Electrical Engineering at Beihang University. Her research interests include mechatronics, modeling and simulation.

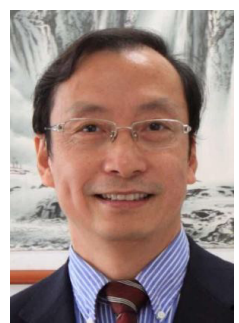

Zongxia Jiao Zongxiao Jiao(M'11-SM'13) received the B.S and Ph.D degrees from Zhejiang University, Hangzhou, China, in 1985 and 1991, respectively. He was with Beihang University, Beijing, China, as a Postdoctoral Fellow from 1991 to 1993 and has been a Professor there since 1994. He is currently the Dean of the School of Automation Science and Electrical Engineering, Beihang University. His research interests include actuators, sensors, fluid power and more electrical aircraft. Prof. Jiao was recipient of the Changjiang Scholar Professor in 2006 and the Distinguished Young Scholar of China in 2008. 